\title{
Multifunctionalization of Nanostructured Metal Oxides
}

\author{
Zijie Yan, ${ }^{1}$ Yu Wang, ${ }^{2}$ Dawen Zeng, ${ }^{3}$ Douglas B. Chrisey, ${ }^{4}$ and Min Liu ${ }^{5}$ \\ ${ }^{1}$ Department of Chemical \& Biomolecular Engineering, Clarkson University, Potsdam, NY 13699, USA \\ ${ }^{2}$ School of Materials Science and Engineering, Nanchang University, Nanchang 330029, China \\ ${ }^{3}$ Department of Materials Science and Engineering, Huazhong University of Science and Technology, Wuhan 430074, China \\ ${ }^{4}$ Department of Physics and Engineering Physics, Tulane University, New Orleans, LA 70118, USA \\ ${ }^{5}$ Department of Electrical and Computer Engineering, The University of Toronto, Toronto, ON, Canada M5S 3G4
}

Correspondence should be addressed to Zijie Yan; zyan@clarkson.edu

Received 16 September 2015; Accepted 16 September 2015

Copyright (c) 2015 Zijie Yan et al. This is an open access article distributed under the Creative Commons Attribution License, which permits unrestricted use, distribution, and reproduction in any medium, provided the original work is properly cited.

Metal oxides are a rich family of materials that have nourished many research areas. From colossal magnetoresistance to multiferroicity and from catalysts to wearable devices, metal oxides never lack new materials with fascinating properties and great potential for device applications. The ability to control materials at the nanometric level has further broadened the landscape of research for metal oxides. With rational control of sizes, structures, compositions, and morphologies, nanostructured metal oxides can possess novel optical, electronic, magnetic, and/or mechanical properties that do not exist in bulk forms. These properties have enabled applications of metal oxides in various areas, including electronics, photonics, sensors, catalysis, photovoltaics, and batteries. A better understanding of the origin of novel metal oxide properties promises further improvements that are waiting to be discovered, yet difficulties still exist for applications that require integrating or balancing multiple aspects of the material properties. The integration of metal oxides in prototype devices needs innovations in synthesis, functionalization, assembly, and characterization of nanostructures, which in turn will inspire new applications of metal oxides.

This special issue features articles that address these challenges. The authors report here their achievements in the design, fabrication, modification, and characterization of multifunctional metal oxide nanostructures. It is worth noting that $\mathrm{YBa}_{2} \mathrm{Cu}_{3} \mathrm{O}_{7-x}$ (YBCO) is a well-known material for "high temperature superconductivity." However, in the paper entitled "Photocatalytically Active $\mathrm{YBa}_{2} \mathrm{Cu}_{3} \mathrm{O}_{7-x}$ Nanoparticles Synthesized via a Soft Chemical Route," Z. Shen et al. explored the chemical properties of YBCO and found that YBCO nanoparticles could show excellent photocatalytic effects without compromising their normal superconductivity properties. This work provides a good example of obtaining multifunctional metal oxides based on the surface effects of nanostructures.

In another article entitled "Nanoscale Ferroelectric Switchable Polarization and Leakage Current Behavior in $\left(\mathrm{Ba}_{0.50} \mathrm{Sr}_{0.50}\right)\left(\mathrm{Ti}_{0.80} \mathrm{Sn}_{0.20}\right) \mathrm{O}_{3}$ Thin Films Prepared Using Chemical Solution Deposition," V. S. Puli et al. investigated the ferroelectric/piezoelectric effects of $\left(\mathrm{Ba}_{0.50} \mathrm{Sr}_{0.50}\right)\left(\mathrm{Ti}_{0.80} \mathrm{Sn}_{0.20}\right) \mathrm{O}_{3}$ (BSTS) thin films at the nanoscale level using piezoresponse force microscopy and revealed the nanoscale switchability of ferroelectric polarization in these chemical solution deposited BSTS thin films. This observation opens up the possibility of further optimizing physical and electrical properties of BSTS films for practical applications, including nonvolatile ferroelectric memories, data-storage media, piezoelectric actuators, and electric energy storage capacitors.

The guest editors hope that the special issue will draw attention to the multifunctionalization of nanostructured metal oxides and stimulate not only the discovery of new multifunctional metal oxides, but also the detection of new properties in traditional metal oxides via the state-of-the-art nanotechnology.

Zijie Yan

Yu Wang

Dawen Zeng

Douglas B. Chrisey

Min Liu 

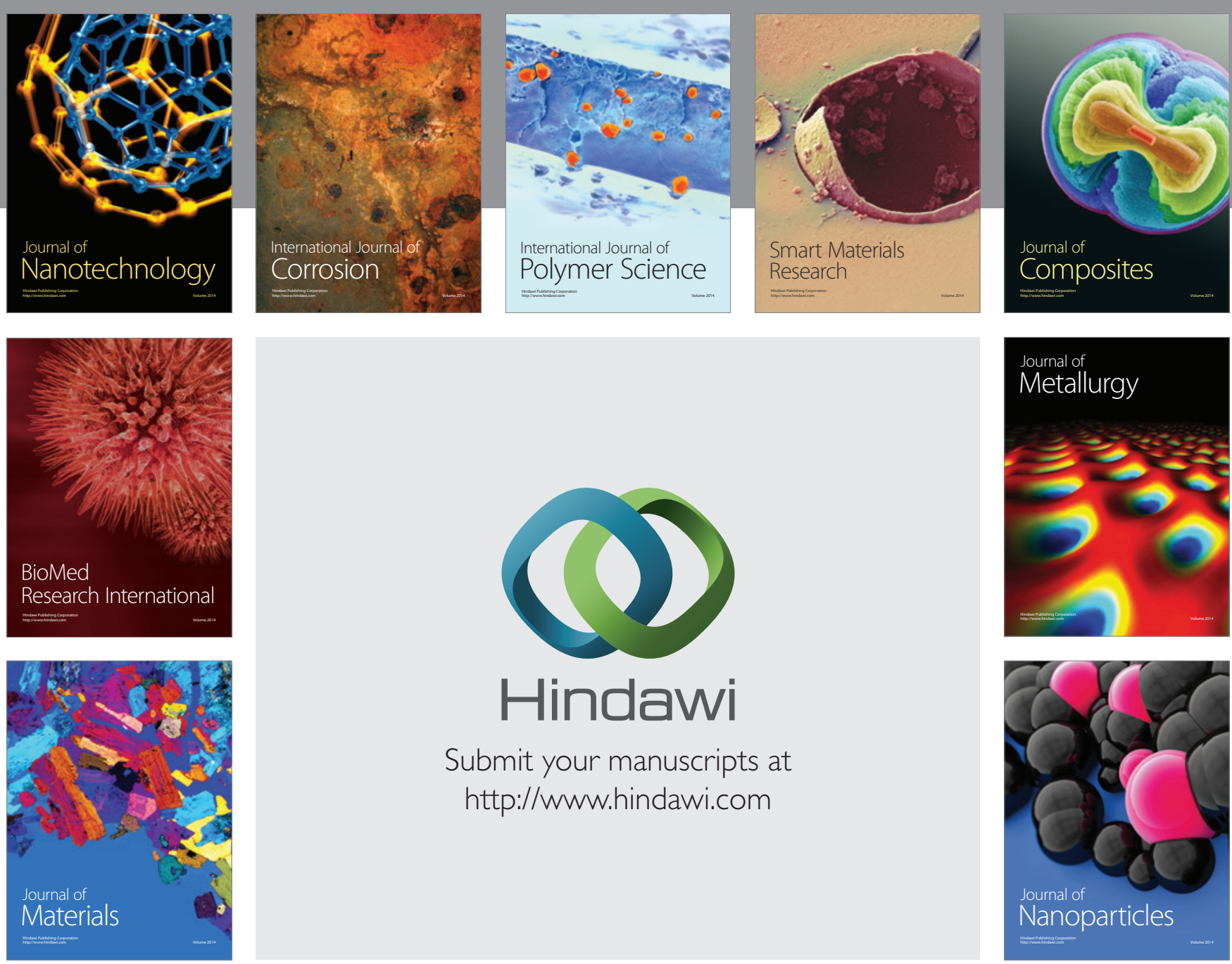

Submit your manuscripts at http://www.hindawi.com
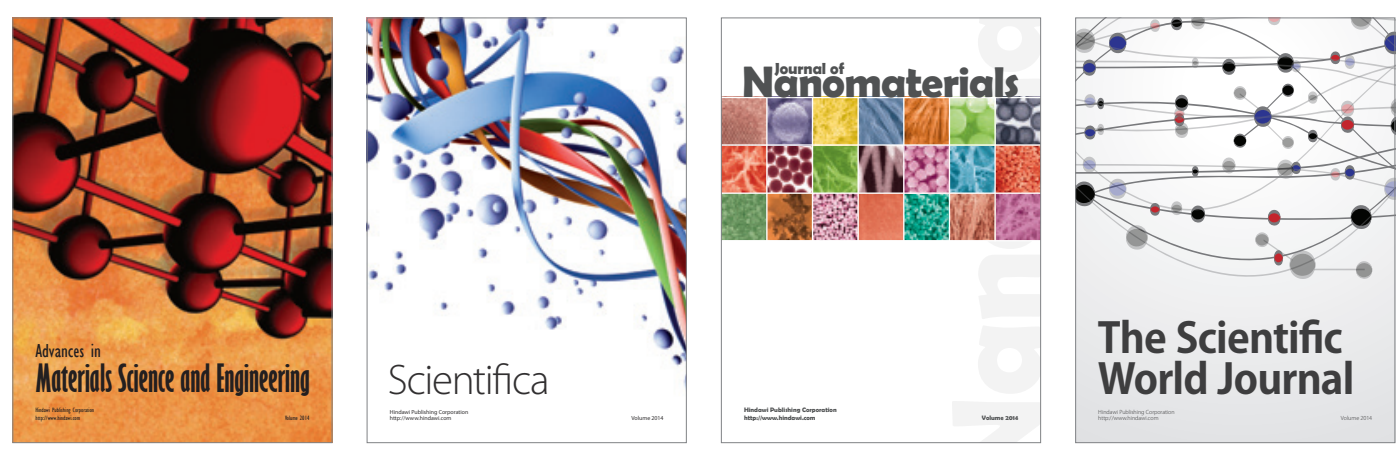

\section{The Scientific World Journal}
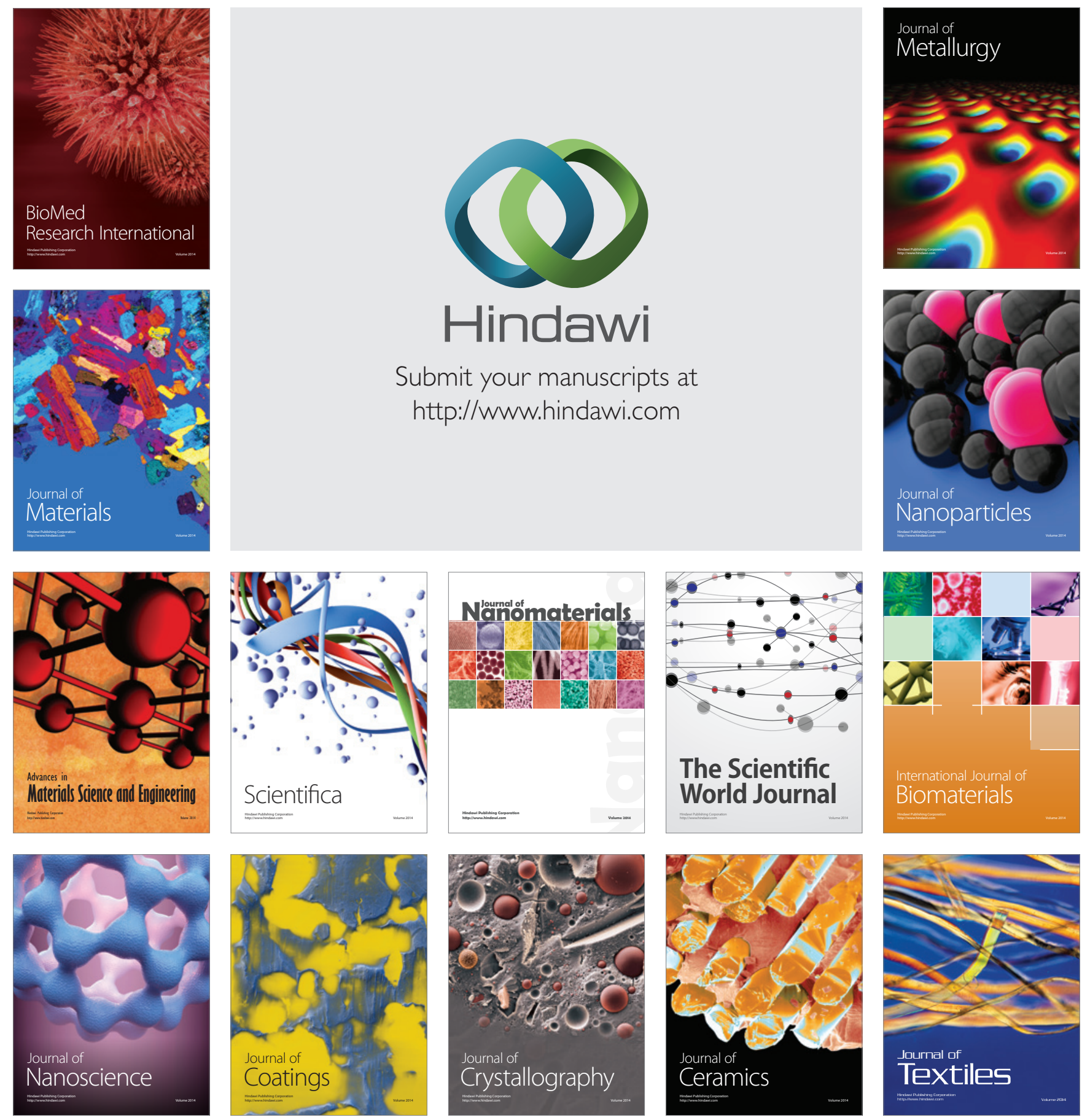